\title{
Innovative Sketch Board Mining for Online image Retrieval
}

\author{
Huda Abdulaali abdul Baqi ${ }^{1,4}$, Ghazali Sulong ${ }^{1,2}$, Siti Zaiton Mohd Hashim ${ }^{3} \&$ Zinah S.Abdul jabar ${ }^{4}$ \\ ${ }^{1}$ UTM-IRDA Digital Media Centre (MaGIC-X), Faculty of Computing, Universiti Teknologi Malaysia, Malysia \\ 2 UMT. School of Informatics and applied mathematics, Universiti Malaysia Terengganu, 21030 Kuala \\ Terengganu Malysia \\ ${ }^{3}$ Big Data Centre, UTM Skudai Johor Takzin, Malaysia \\ ${ }^{4}$ University of Al -Mustansiriyah, College of Science, Computer Dep., Baghdad, Iraq \\ Correspondence: Huda Abdulaali abdul Baqi, UTM-IRDA Digital Media Centre (MaGIC-X), Faculty of \\ Computing, Universiti Teknologi Malaysia, 81310 Skudai, Malaysia. E-mail: hudaabdulaali2015@gmail.com
}

Received: October 5, 2016

Accepted: October 22, 2016 Online Published: November 22, 2016

doi:10.5539/mas.v11n3p13

URL: http://dx.doi.org/10.5539/mas.v11n3p13

\begin{abstract}
Developing an accurate and efficient Sketch-Based Image Retrieval (SBIR) method in determining the resemblances between the user's query and image stream has been a never-ending quest in digital data communication era. The main challenge is to overcome the asymmetry between a binary sketch and a full-color image. We introduce a unique sketch board mining method to recover the online web images. This image conceptual retrieval is performed by matching the sketch query with the relevant terminology of selected images. A systematic sequence is followed, including the sketch drawing by the user in interpreting its geometrical shape of the conceptual form based on annotation metadata matching technique achieved automatically from Google engines, indexing and clustering the selected images via data mining. The sketch mining board being built in dynamic drawing state used a set of features to generalize sketch board conceptualization in semantic level. Images from the global repository are retrieved via a semantic match of the user's sketch query with them. Excellent retrieval of hand-drawn sketches is found to achieve the recall rate within 0.1 to 0.8 and a precision rate is 0.7 to 0.98 . The proposed technique solved many problems that stat-of-art suffered from SBIR (e.g. scaling, transport, imperfect) sketch.

Furthermore, it is demonstrated that the proposed technique allowed us to exploit high-level features to search the web effectively and may constitute a basis for efficient and precise image recovery tool.
\end{abstract}

Keywords: sketch-based retrieval, image retrieval, shape representation, annotation based semantic matching

\section{Introduction}

Previously, there has been a wide interest and progress on computer aided retrieval of media data. The advances in this area have allowed users to look for a multimedia object in large repositories in a more efficient way. As advances in multimedia retrieval, increase, new interesting, challenging applications are coming up. Image retrieval has very important applications (Banfi, F., 2000) that are beyond the traditional application based on searching such as Architecture and Interior Design, Biochemical, Education,...etc.., for that the easy way to retrieving images is by means textual metadata describing the object in the image. However, then retrieved by text not always come with reliable human tags. Although, many authors have addressed the several methods have been invented (Funkhouser et al., 2003, Eitz, et al., 2012). For example, an easy way to express the user query is by using a line-based hand-draw. One of the current interesting applications is an input draw (sketch)

Definition: a sketch consists of a group of elements; a sketch is represented as a structure of a shape used to find images relevant to this shape leading to a method of sketch-based image.

The sketch based image retrieval (SBIR) is part of the image retrieval field. In an SBIR system, the input is a simple sketch representing one or more objects. As shown in Figure 1. 


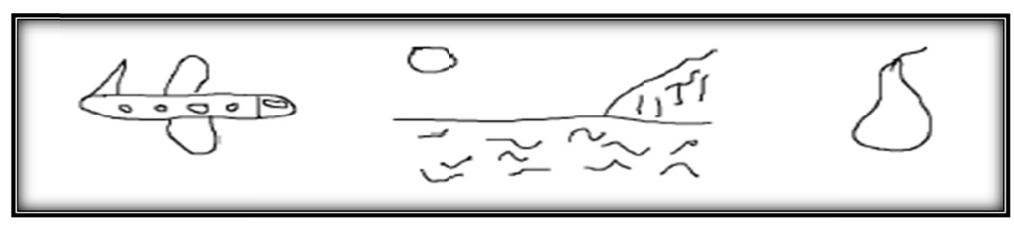

Figure 1. Sketch query example

The retrieved images based on sketch technique is studied from several areas to get the retrieval of images accurately and fast and find out many methods to retrieved images from limited data base or small database and satisfy a good result, but still not satisfy good retrieved from large database to be trying to reach to retrieve images from large database reach to retrieve from the web. A precise image conceptual retrieval scheme for online sketches is far from being achieved. Furthermore, unlike other classical methods to detect shapes similar to the present sketch, the proposed technique avoids the efficiency issues regarding shape detection of low-level features and extracts them by for example, the Hough transform or canny (Dube and Zell, 2011; Abdulbaqi et al., 2014). In this communication, we report a novel method to input sketch and retrieved images based on the sketch online. This method is based on a different feature called the keyshape mining of the visual objects and used the semantic structure to achieve the name of sketch (metadata) to retrieved images that is yields a significant improvement in the retrieval effectiveness. This paper is organized as follows; Section 3 methodology of online SBIR. Section 4 presents Sketch interpretation. Section 5 Drawing question sketch online. Section 6 Semantic Matching. Section7. Result and desiccation. Section 8 concludes the paper.

\section{Related Work}

Although many authors have been involved in content based image retrieval using an example image as a query, some relevant works on sketch based image retrieval have only appeared in the last three years. It is established that an easy way to communicate the user query is through a line-based hand-drawing (a sketch), which leads to the sketch-based image retrieval (SBIR). Only few studies have addressed the sketch based image retrieval methods. Some of the reports work on Edge Histogram Descriptor (EHD) (D.k et al., 2000) by extracted information, in the form of feature vectors, is clustered to form a codebook that is then used under the Bag Of Features (BOF) approach. This work is also relevant because the authors propose the first system to build a benchmark for the SBIR problem. One outstanding property of this benchmark is that it takes into account the user opinion about the similarity between sketches and test images. This is highly important because the ultimate goal of a retrieval system is to satisfy the user requirements. Recently, ( Saavedra and Bustos, 2014) proposed a procedure to identify a sketch in which the structural components are obtained and the sketch is disintegrated into a set of basic geometric shapes this a new method fined the spatial relationship between these basic geometric shapes eventually leads to the sketch identification, this methods Known as 'keyshape', it also to enhance the effectiveness of the mechanism for efficient retrieval of sketches, the proposed method combines the 'keyshape' with a Bag of Features (BOF). In fact, a sketch is based on related works the techniques are used to recreate images based on a sketch query by drawing only the most important edges to try to paint an image that looks like the one that is being sought consisting of the contour of one objects in the image. Other methods extract features of Histogram of Gradients and discrete distance transform (Tencer et al., 2012), (Slong et al.,2015) introduced a comparative study to find out which method, dose the image appearing after processing look like a sketch in matching. (Parui and Mittal ,2014) Has displayed the management of each image in the database through pre-processing in order to obtain the image, extract the sequences and contour segmentation to enable partial matching of chains by presenting a comparative study to find which method results in images most representative of the sketch. (Cao et al., 2011) Developed a system that supports query-by-sketch in a large database, (two billion images). They chose a raw edge pixel as representation and Chamfer matching as the matching mechanism for their system. The system is composed of two major parts, offline image processing, and online image retrieval. First, content-based image retrieval (CBIR) technology is used to retrieve a set of visually similar images from a large-scale web image set. However, it is still a challenge to use this method with a sketch to retrieve image annotation. Some challenging problems such as image annotation were studied in a data-driven way.

\section{Methodology}

The proposed technique consists of three stages. First, sketch board mining that allows us to draw the sketch online and mining the important point of the sketch then claster the keyshape. Second, interpretation sketch drawn by finding a Spatial relationship between keyshaps depended on centrude point of the object. Third, match the 
concept (metadat) of the sketch selectad with annotation metadata in the image repository online. Fig.2. Illustrates the schematic of our research framework.

\subsection{Sketch Board Mining}

The proposed online sketch board mining system consists of three main components including canvas drawing board, a set of geometric keyshape mining and interpretation of drawing sketch. The drawing board resembles an online sketch editor that is used to allow the users to draw a sketch in real time using mouse clicks with all the mouse events recorded. The geometric shape elements are created to interpret various types of geometrical shapes (keyshape) of the sketch based on the recorded mouse events. For each object, a certain number of sketches are saving as templates to represent the object. The templates are then stored in a database as a semantic space known as the template sketch.

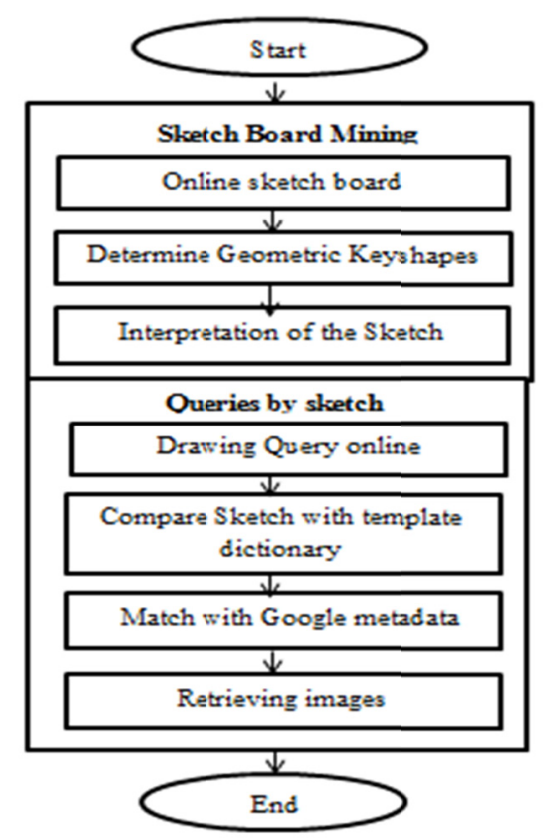

Figure 2. General framework of the proposed methodology

\subsection{Online Sketch Board}

The sketch board mining system, which is based on keyshape provides important information of a sketch such as start, end, middle and centroid points. These key points are used to determine the attributes and to mine the elements (key shape) of the sketch, which include straight lines, curves, ellipses and circles. The keyshapes are then used to interpret the sketch. In this study, a sketch drawing board, which is developed using HTML5 canvas and JavaScript, acts like a sketch editor (see Figure 3). It provides important features, among others: (1) It can connect directly to the Internet (2) It supports latest browsers, including Chrome, Firefox and Opera (3) It can receive input via mouse click and keyboard, and (4) The user can freely draw any kind of sketch.

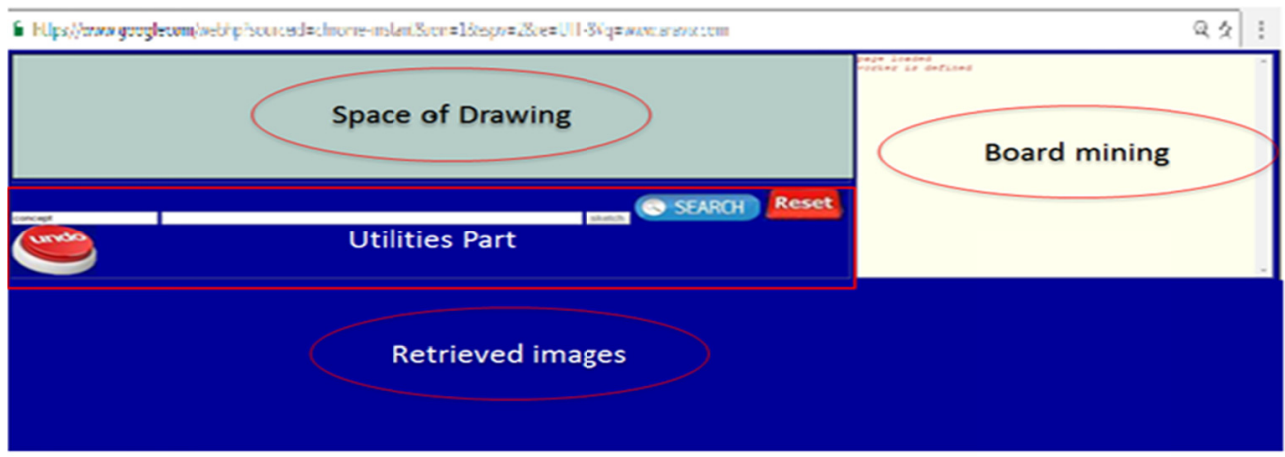

Figure 3. The sketch board editor 
The main function of the proposed online drawing board is to allow a user to draw a sketch using a mouse and to capture all mouse events. The events incluMouseDown, MouseUp and MouseMove. MouseDown indicates the starting point of an element/attribute of the sketch, MouseUp denotes the ending of the element and MouseMove represents the events in-between the MouseDown and MouseUP of the element. For every single element of the sketch, the system provides a unique ID and records all the mouse events in terms of their coordinates. Figure 4 depicts a complete sequence of events of a sketch along with its captured data.

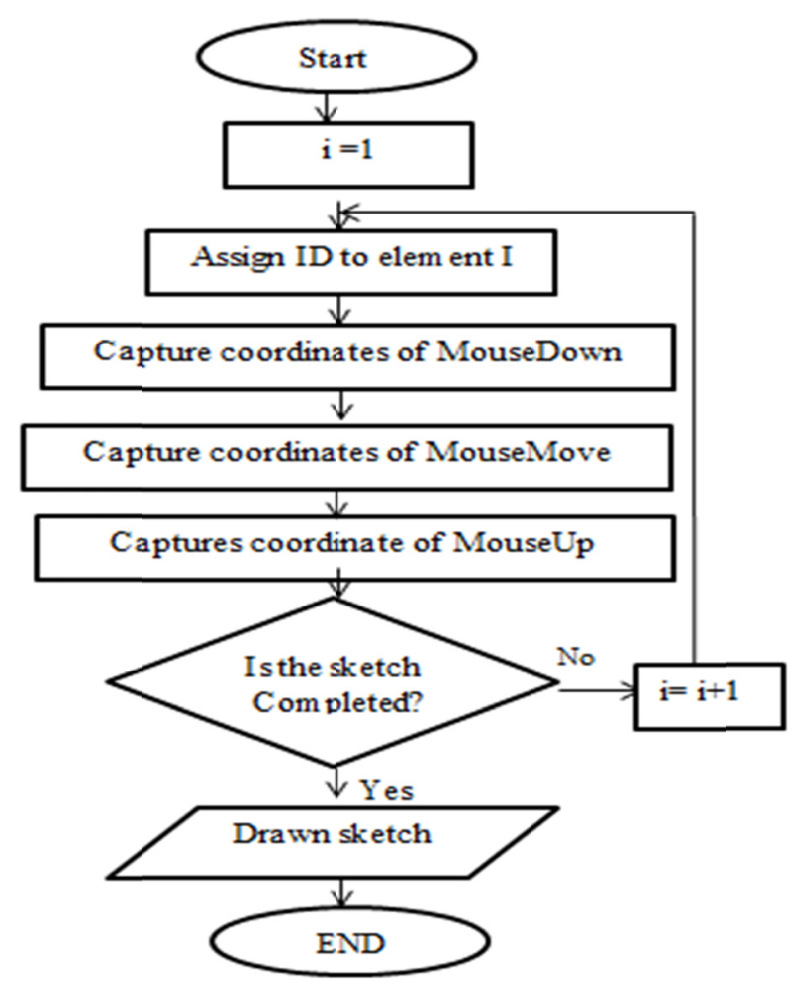

Figure 4. A block diagram of data acquisitions by the sketch board mining during a sketch drawing session

\subsection{Determining Geometric Keyshape}

In this study, data mining method is used to specify three basic geometric keyshapes. Mining is a data knowledge discovery that represents the process of analysing data from different perspectives in order to find the useful information (Aggarwal, 2007). It is the analytical tool that is used in analysing the data coming from sketch board including many different dimensions or angles with the aim of categorizing it and identifying the relationships. It also finds the correlations and template in large relational query databases. In this case, the drawn object will be classified into separated elements that represent the shape features based on canvas input representation and supported by the specific design of data mining.

Definition 1: A keyshape is a simple geometric shape that is composed of more complex elements. Examples of a key shape may be a circle, an ellipse, a line, among others (Saavedra and Bustos, 2014).

Definition 2: Mining is the process of analysing data from different perspectives and summarizing the identified relationships into useful information (Gupta et al., 2011).

Thus, we propose an efficient strategy that is exploiting the structural information provided by sketch depending on the basic geometric elements that can be detected by mining in a method known as "keyshape mining". Keyshape mining is a geometrical shape detected by mining the string of pixels like (circle, line, and curve) to determine eleven types of geometric elements that are given in Figure 5 These elements are then correlated with other simple geometric shapes, thereby creating the element group under a specific concept depending on the elements component and position. Keyshape mining technique needs a new input method that can enable the analysis of the elements based on mining to understand the sketch and to characterize these images by mining the keyshape. 


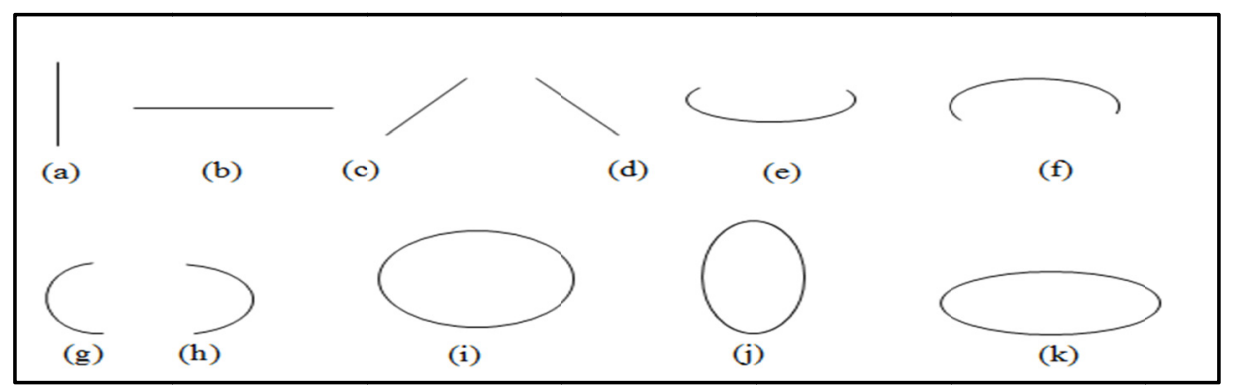

Figure 5. The eleven element types (a) horizontal line, (b) vertical line, (c) northward line, (d) southward line, (e) northward curve, (f) southward curve, (g) eastward curve, (h) Sketch westward curve, (i) circle, (j) x-axis ellipse, (k) y -axis ellipse

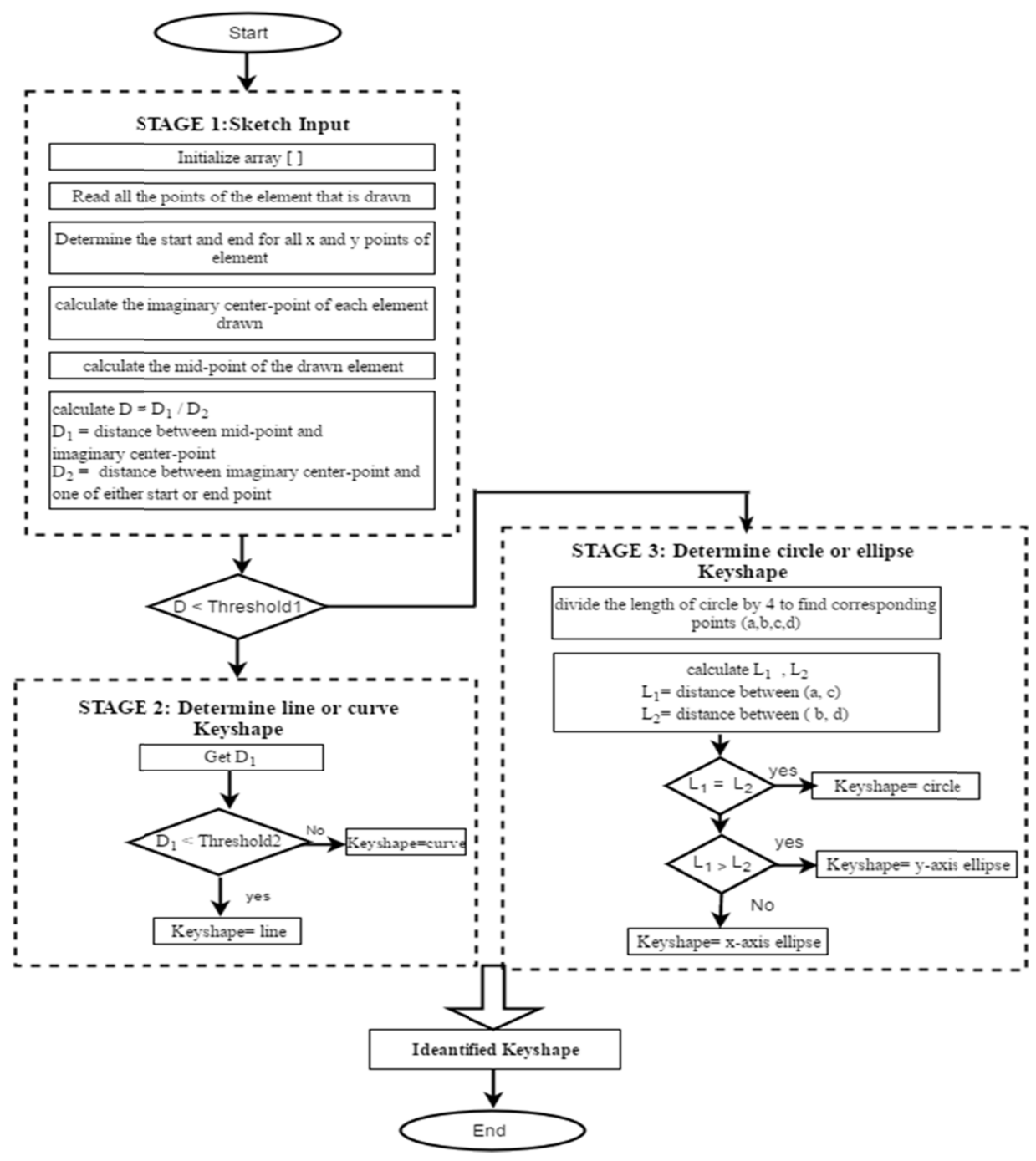

Figure 6. Displays the general framework to determine the drawn keyshape element depending on calculated threshold

Two different thresholds are used. Threshold(1) value is equal to 1 which determines whether the Keyshape is line or circle because the threshold reflect the difference between the imaginary point and the mid- point, fore that the threshold 2 value which equal to 2 reflects that arc generated in the sketch element and its determine as a curve. This framework consists of three stages to determine keyshape element: first, input query to determine the important variables that are used to calculate the keyshape; second, determine if the keyshape is a line or curve; third, determine if the keyshape is circle or type of ellipse. 


\section{Stage 1: Sketch Input}

This stage describes important variables, $\mathrm{x}$ and $\mathrm{y}$, related to each drawn element used to determine the keyshape of the sketch shown in Figure 7. These variables are:

1. Start point $\left(\mathrm{x}_{1}, \mathrm{y}_{1}\right)$ : it is the first point of a drawn element generated by the MouseDown.

2. End point $\left(\mathrm{x}_{\mathrm{n}}, \mathrm{y}_{\mathrm{n}}\right)$ : it is the last point of the drawn element generated by the MouseUp event, where $\mathrm{n}$ denotes the total number of acquired points of the element.

3. Chain point $\left(\mathrm{x}_{\mathrm{i}}, \mathrm{y}_{\mathrm{i}}\right),(\mathrm{i}=2,3, \ldots, \mathrm{m}, \ldots, \mathrm{n}-1)$ denotes the point located in-between $\left(\mathrm{x}_{1}, \mathrm{y}_{1}\right)$ and $\left(\mathrm{x}_{\mathrm{n}}, \mathrm{y}_{\mathrm{n}}\right)$ of the drawn element generated by the MouseMove event.

4. Mid-point $\left(\mathrm{x}_{\mathrm{m}}, \mathrm{y}_{\mathrm{m}}\right)$ : it is the point that lies in the middle of a drawn element will be calculated for each drawn element using equations 1 and 2, respectively. These point lies on the real line that links the start and end points.

$$
\begin{gathered}
x_{m}=\frac{\sum_{i=1}^{n} x_{i}}{2} \\
y_{m}=\frac{\sum_{i=1}^{n} y_{i}}{2}
\end{gathered}
$$

5. Imaginary centre-point $\left(\mathrm{x}_{\mathrm{c}}, \mathrm{y}_{\mathrm{c}}\right)$ : it is the centre-point of an imaginary straight line, which is artificially erected linking the start point and the end point directly.

Distances, D1 and D2, will be calculated for each drawn sketch using equations 3 and 4, respectively

$$
\begin{aligned}
\text { D1 } & =\sqrt{\left(\mathrm{x}_{\mathrm{c}}-\mathrm{x}_{\mathrm{m}}\right)^{2}+\left(\mathrm{y}_{\mathrm{c}}-\mathrm{y}_{\mathrm{m}}\right)^{2}} \\
\text { D2 } & =\sqrt{\left(\mathrm{x}_{\mathrm{c}}-\mathrm{x}_{1}\right)^{2}+\left(\mathrm{y}_{\mathrm{c}}-\mathrm{y}_{1}\right)^{2}}
\end{aligned}
$$

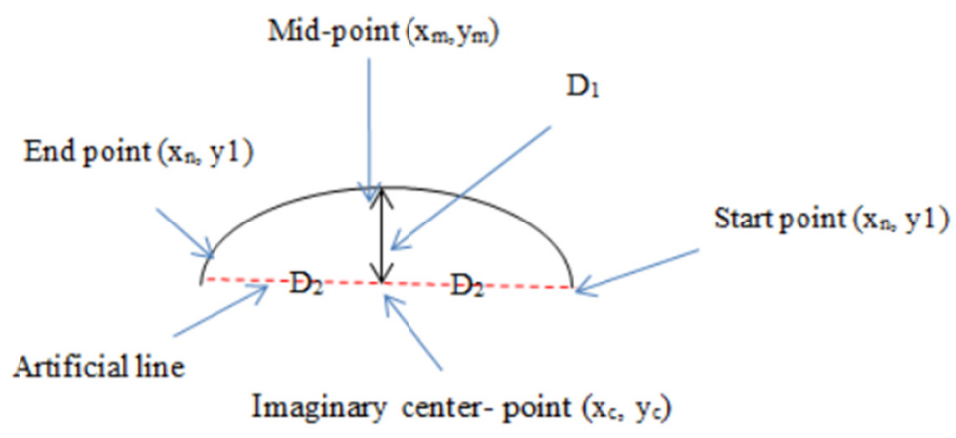

Figure 7. The important points of each drawn element

Where

$\mathrm{D}_{1}=$ distance between imaginary centre point and mid-point.

$\mathrm{D}_{2}=$ distance between imaginary centre point and one of either start or end point.

Depending on the ratio between $D_{1}$ and $D_{2}$, the drawn keyshape element will be classified into one of two groups. The first group represents the circle keyshape and the second represents the line or curve keyshape.

If $\left(D_{1} / D_{2}\right)$ is greater than a specific threshold1, then the keyshape is marked as a circle; otherwise it is a line or curve.

\section{Stage 2: Determination of line or curve keyshape}

Since most of the lines are not drawn in a straight line and sometimes resemble a curve, it is necessary to precisely determine their true nature, line or curve. From multi input for each sketch case, it is determined that the value of Threshold2 (distance between the imaginary centre point and midpoint of the drawn element) can be used to differentiate the line from the curve. For that reason, we need a separate algorithm to determine such problem (see Algorithm 1). 
Algorithm 1: Determination of line or curve keyshapes

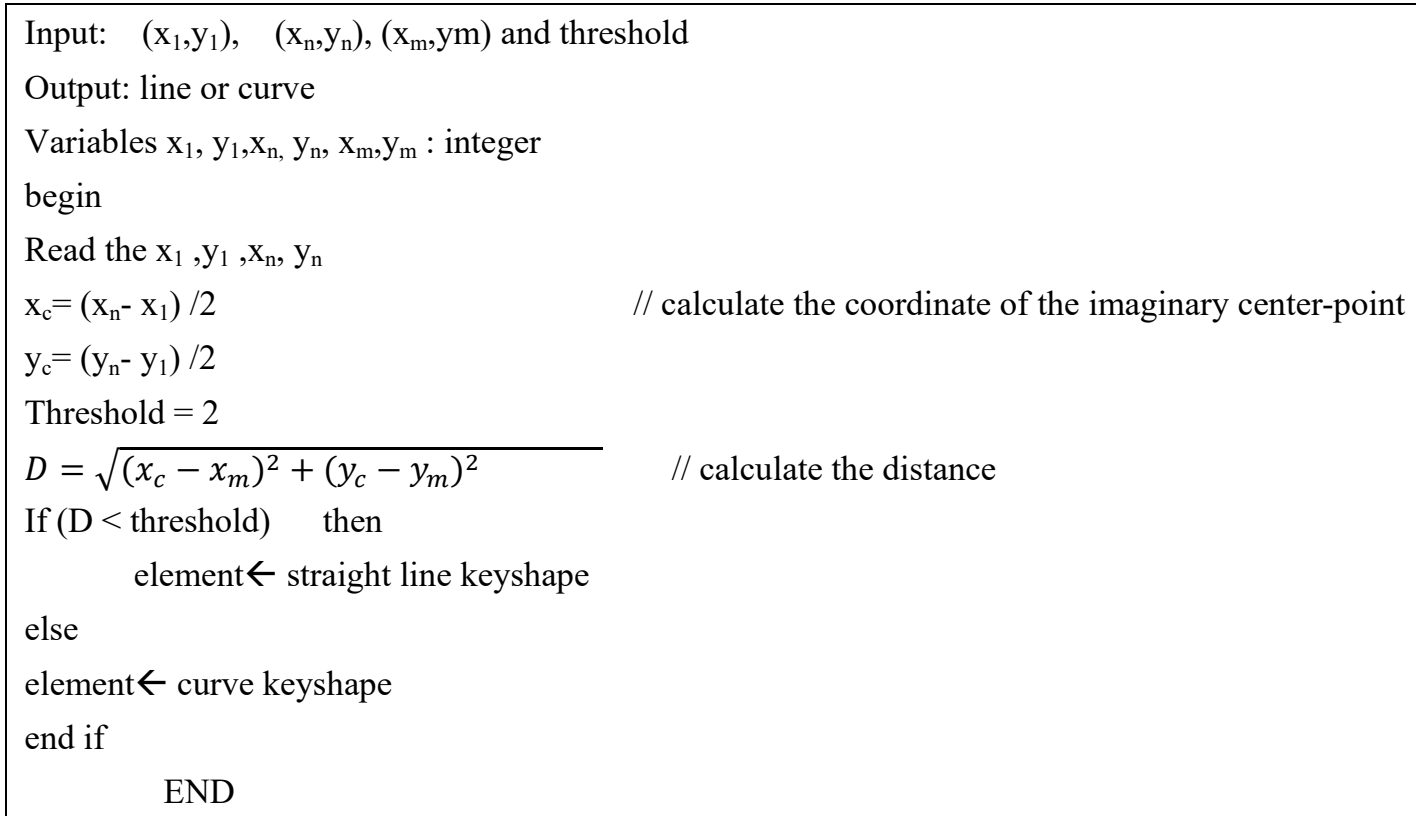

Algorithm 2 is used to determine the type of the straight line, depending on differences between the corresponding coordinates value of start and end points that are represented in $\Delta \mathrm{x}$ and $\Delta \mathrm{y}$ where:

$\Delta \mathrm{X}=\left(\mathrm{x}_{\mathrm{n}}-\mathrm{x}_{1}\right), \Delta \mathrm{Y}=\left(\mathrm{y}_{\mathrm{n}}-\mathrm{y}_{1}\right)$

Algorithm 2: Determination of various line type keyshapes

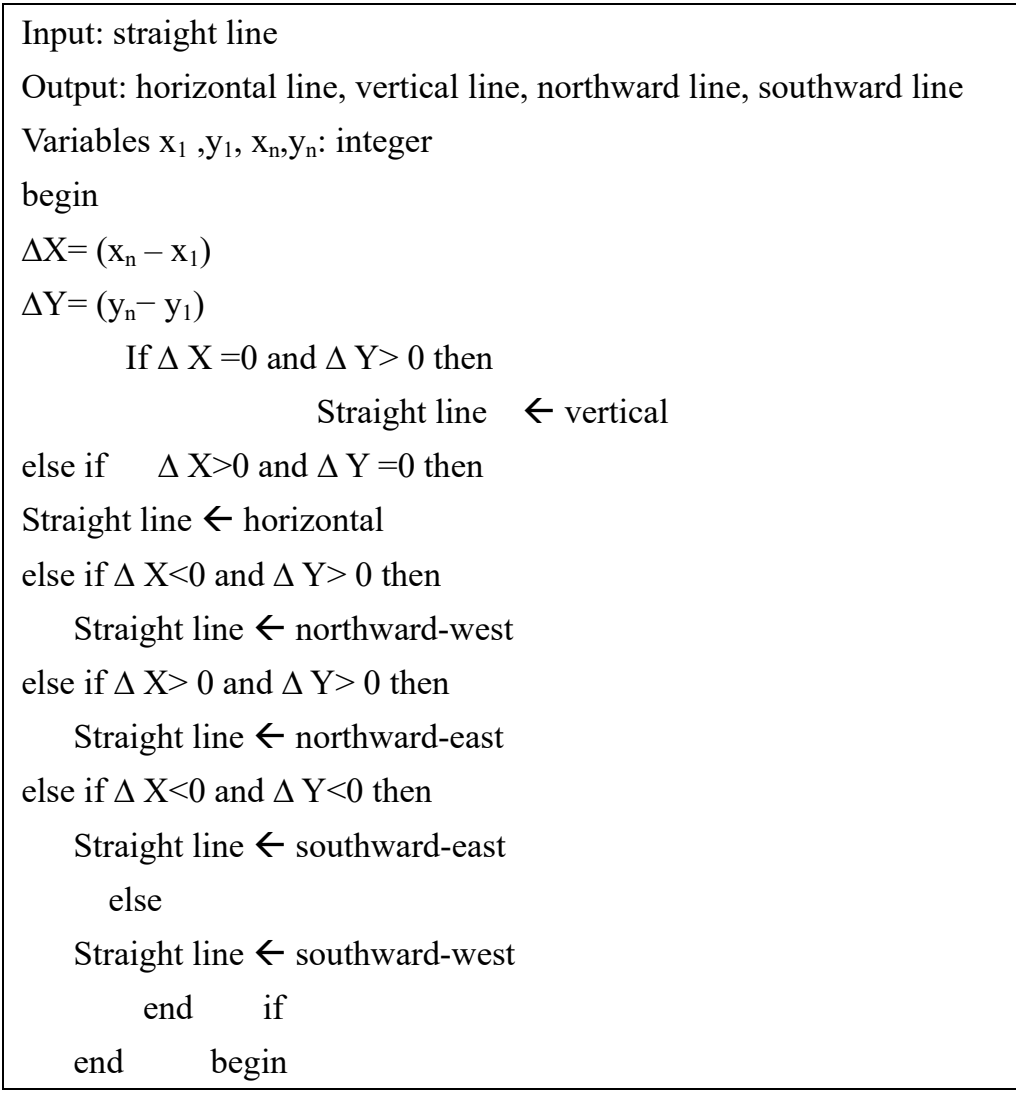




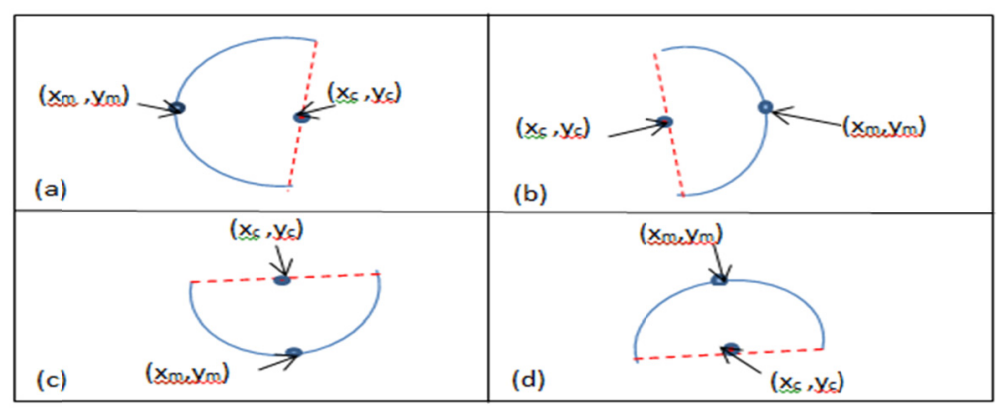

Figure 8. Four types of curve (a) westward curve, (b) eastward curve, (c) southward curve, and (d) northward curve

In curve keyshape situations, the direction of the curve depends on the coordinates of both midpoint and imaginary points. Generally, there are four curve types, westward, eastward, northward and southward. In the first two cases, the direction of the curve depends on the dominanting value of X-coordinate, while in the last two cases, the curve direction depends on the dominanting value of Y-coordinate. Figure 7 represents all types of curve along with Algorithm.3.

Algorithm 3: Computation of curve types

Input: drawn curve
Output: curve types
Variables $\mathrm{x}_{\mathrm{m}}, \mathrm{x}_{\mathrm{c}}$ : integer
begin
If $\mathrm{x}_{\mathrm{m}}>\mathrm{x}_{\mathrm{c}}$ then
Key shape $\leftarrow$ west curve
else
keyshape $\leftarrow$ eastward curve
end if
If $\mathrm{y}_{\mathrm{m}}>\mathrm{y}_{\mathrm{c}}$ then
Keyshape $\leftarrow$ north curve
else
keyshape $\leftarrow$ southward curve
end if
end begin

\section{Stage 3: Determine circle or ellipse keyshape}

In this stage, the keyshape will be determined as a circle or ellipse according to Figure 9 (a) - (c). By implementing the following steps as illustrated in Algorithm 4.

i) For each drawn element, four basic points (start, end, midpoint and imaginary midpoint points) are used in Equation (3) to determine the circle and ellipse.

$$
\text { Circle }=\left(\sqrt{\left|x_{c}-x_{m}\right|^{2}+\left|y_{c}-y_{m}\right|^{2}}-\sqrt{\left|x_{n}-x_{1}\right|^{2}+\left|y_{n}-y_{1}\right|^{2}}\right.
$$

ii) Evenly divide the length of the drawn circle by 4 to find four points, which are termed as a, b, c and d. 
iii) Calculate the distance (L1, L2) using Algorithm 4, where L1 is the distance between (a and c), while L2 is the distance between ( $b$ and d). These two distances are used to differentiate between circle and ellipse:

If $(\mathrm{L} 1=\mathrm{L} 2)$ then the keyshape is circle, otherwise ellipse. If $(\mathrm{L} 1>\mathrm{L} 2)$ then the keyshape is $y$-axis ellipse, else $\mathrm{x}$-axis ellipse.

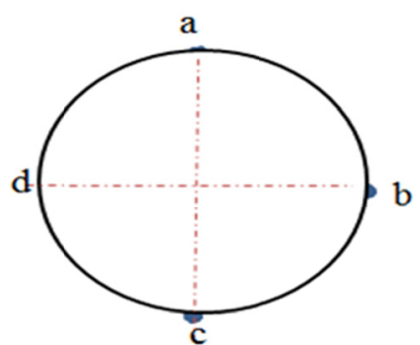

A

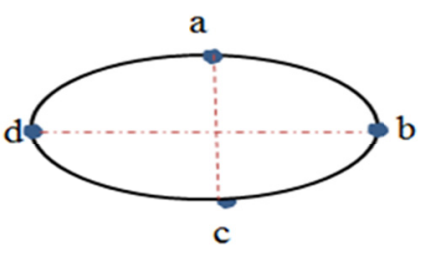

B

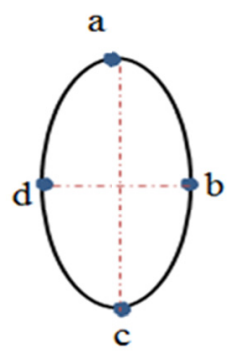

C

(a)

(b)

(c)

Figure 9. Three types of Keyshape (a) circle, (b) x-axis eastward ellipse, (c) y-axis westward ellipse

Algorithm 4: Determine circle or ellipse

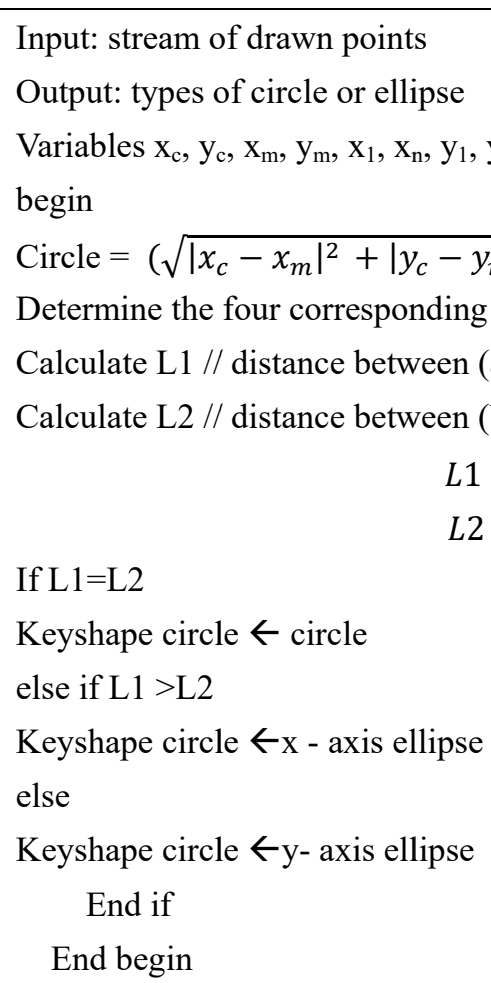

After determining the type of the element, the other issue in this step is to calculate

The angle of each element based on Cartesian rule (see Equation 4). The benefit of knowing the angle is to specify the element slope with regard to the canvas drawing area. Figure 10 presents an example of two drawn elements with their respective angle.

$$
\theta=\frac{\tan ^{-1}\left(\mathrm{y}_{\mathrm{n}}-\mathrm{y}_{1}\right)}{\left(\mathrm{x}_{\mathrm{n}}-\mathrm{x}_{1}\right)}
$$




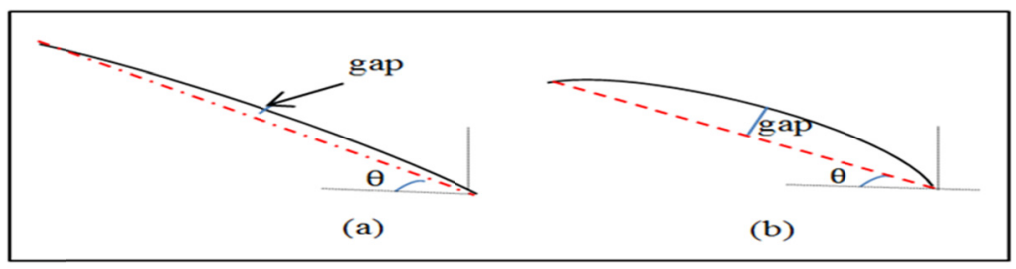

Figure 10. Angle of element

Depending on the (start, end and imaginary midpoint) points of the keyshape element, a basic mathematical formula is used to find the spatial relationship between these elements depending on the direction of each element in relation to another in the same sketch. The centroid of the object (sketch) also represents a very important point related to the object. It is known that each object has a certain centroid. This means that the centroid can represent one of the distinguished points to the object. From this point of view, the centroid adds a new value to the object that makes it unique because the element's position related to centroid point will specify the object element details, meaning that the centroid direction represents the uniqueness of the sketch (see Equation 5 and Equation 6). It is this point that paves the way to specifying an object's name for each sketch.

The equations of the centroid calculation for (n) elements in the sketch (Boolos et al.; 2002; Mendelson, 2009) are:

Where $\bar{X}$ : Centroid $\mathrm{x}, \bar{Y}$ : centroid $\mathrm{y}$ and $\mathrm{n}$ is number of elements.

$$
\begin{aligned}
& \bar{X}=\sum_{i=1}^{n} x_{i} / \mathrm{n} \\
& \bar{Y}=\sum_{i=1}^{n} y_{i} / \mathrm{n}
\end{aligned}
$$

\section{Sketch Interpretation}

Interpretation is a technical notion that approximates the idea of representing a logical structure inside another structure (Zhou et al., 2012; Bozas and Izquierdo, 2012). It provides a set of basic geometrical/direction primitives and allows for the addition of more domain-specific semantic relations. The main challenges in interpretation technique would be to firstly discover the components that the sketch is composed of, and secondly, what these components of the sketch actually mean. In other words, the interpretation can be separated into sketch element classification and recognition the sketch object.

In this novel technique, the designed sketch board interprets the element of the structural components by mining the set of points drawn on the sketch board to identify the keyshape elements of their basic geometrical class. Then, these keyshapes are conceptualized to obtain their specific meaning of the sketch based on the user application. This specific meaning represents the type of the sketch (template) that will be collected from all the training sketches, and the more common template will be the dependent template used in retrieving images based on this template sketch. This step basically depends on the spatial relationship between the keyshape of the drawn sketch.

Spatial relationships explore the concept of where the element are in relationship to other elements in the same object (Guarino and Welty, 2000). The study of spatial relationship of the elements is the core of the sketch meaning recognition because it specifies the unique structure of the sketch based on unique correlations between the object elements.

This step provides the correlation between the interpreted keyshape sketch elements and tries to find spatial relationships between this element of object $\left(\mathrm{ID}_{1}, \mathrm{ID}_{2}, \mathrm{ID}_{3, \ldots . .} \mathrm{ID}_{\mathrm{n}}\right.$, where $\mathrm{ID}$ is the element type identified from determining the geometric keyshape elements sketch. According to this study, the interpretation process is a high-level stage that recognizes the sketch meaning depending on spatial relationship between the keyshape elements. For instance, when a person is asked to make a simple drawing of a teapot, he or she will probably draw only three components: the body, the spout and the handle. The interpretation used in this work formally represents the sketch object assignment based on the spatial relationships that come from the content of object elements correlated with the direction of each element such as (northward-west, southward-east, southward-west, southward-east) among these elements depending on the distance between a centroid of the main shape and the imaginary centre point of all elements of the sketch. Figure 11 shows how to specify the direction of each element of the sketch based on the imaginary point of each element correlated with centroid point of object (sketch). It is clear that when an imaginary point takes place in the right position of the centroid point as in point 
(1), it will specify that this element occurs Northward-east of centroid point and the others will be read in the same way.

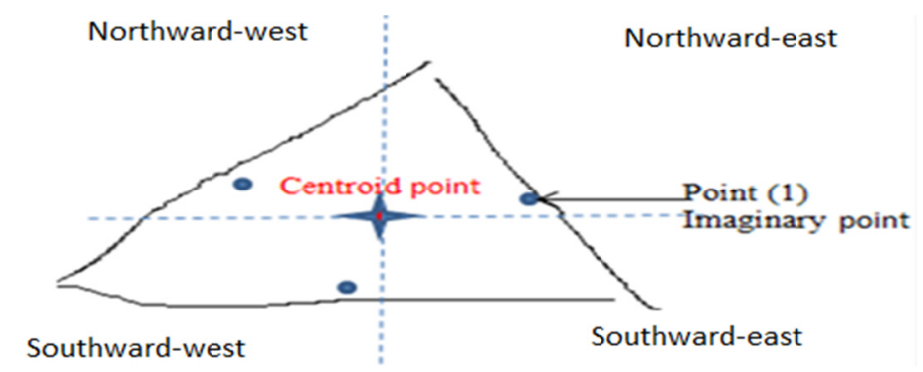

Figure 11. Specifying the direction of each element based on an imaginary correlated with centroid point

The sequences of operations to find the spatial relationships are described below:

1. Detect the imaginary center points of keyshape (geometric element).

2. Determine the object centroid using Equations 5 and 6 .

3. Count the number of keyshape elements based on the type of each element as specified in Algorithm (1 to 4), and the angle direction for each element using Equation 4.

4. Determine the distance between the imaginary center points of the element with centroid points of the object based on the direction of the element with respect to angular direction as explained briefly in Algorithm 5.

5. Store the element by (ID type geometry, direction).

The procedures to identify spatial relationship between the keyshape are illustrated in Algorithm 5 .

Algorithm 5: Identify spatial relationship between keyshape types

$$
\begin{aligned}
& \text { Input : } \mathrm{T}_{1} \text { array of string geometric keyshape has a specific ID type } \\
& \text { Output : } \mathrm{T}_{2} \text { array of each input keyshape with corresponding direction } \\
& \text { variables } \mathrm{x}_{1}, \mathrm{x}_{\mathrm{n}}, \mathrm{y}_{1}, \mathrm{y}_{\mathrm{n}}, \mathrm{n}: \text { integer } \\
& \text { begin } \\
& \begin{array}{l}
\mathrm{x}_{\mathrm{c}}=\left(\mathrm{x}_{\mathrm{n}}-\mathrm{x}_{1}\right) / 2 \\
\mathrm{y}_{\mathrm{c}}=\left(\mathrm{y}_{\mathrm{n}}-\mathrm{y}_{1}\right) / 2
\end{array} \\
& \begin{array}{c}
\text { determine object centroid } \\
\text { for } \mathrm{i}=1 \text { to } \mathrm{n} \text { of element }
\end{array} \\
& \begin{array}{l}
\theta=\tan { }^{-1} \frac{y}{x} \\
\mathrm{~T}_{2}(1, \mathrm{i})=\mathrm{T}_{1}(\mathrm{i})
\end{array} \\
& \mathrm{T}_{2}(2, \mathrm{i})=\theta \\
& \text { end for }
\end{aligned}
$$

Based on determined the type of keyshape and identify spatial relationship between these keyshape types as proposed Algorithms 1 to 5, many obstacles in identifying sketch have been addressed including, imperfect sketch, Scaling ( different size of the same sketch drawn), translation (different location of the same sketch). The following example, given in Figure 12, shows different sketches of input (teapot) sketch. The successive steps of 
the proposed method to determine the teapot for six different teapot sketches are as follows:

Step 1: For each sketch, the imaginary centre point of each element (red dots) is recorded using Algorithm (1),

Step 2: For the whole object (each sketch), the centroid point (yellow point) is recorded using Equations 5 and 6.

Step 3: By using the same rules used in Algorithm 2, we applied these rules for the whole object based on the centroid of the object and each imaginary centre point of element to specify the type of each element (northward curve, $\mathrm{x}$ - axis ellipse, westward curve, westward curve, northward -east line, northward-east line, and y-axis ellipse).

Step 4: As a result, from step 3, a vector consisting of a sequence of attributes (northward curve, $x$ - axis easwardt ellipse, westward curve, westward curve, northward-east line, northward-east line, and y-axis westward ellipse) that describe the elements of the sketch is created depending on the sequence of attribute drawing.

The next subsection presents a detailed explanation of how each obstacle is resolved.

\subsection{Imperfect Sketch}

Imperfect sketch is a disjointed drawing - sketch with disjoint element(s) that is common due to human behaviour. However, this natural vagueness and imprecision of sketches (Weinbrenner et al., 2012). This reflect the different skills of users concerning with the age of the drawer, the behaviours etc. (McManus et al., 2010). From these definitions, we can recognize that drawings of the same sketch may appear as different types depending on the skill of the participant as presented in Figure (12a). The Figure observes different types of drawings of the same object. This problem can be solved by calculating the number of elements correlated with the centroid object which represents the object unity. Figure 11b shows the incomplete sketch that is successfully recognized by our method as a teapot because the numbers of elements connected with the centroid object are the same attributes related to the teapot sketch despite drawing disjointed element as shown in Figure $12 \mathrm{~b}$.
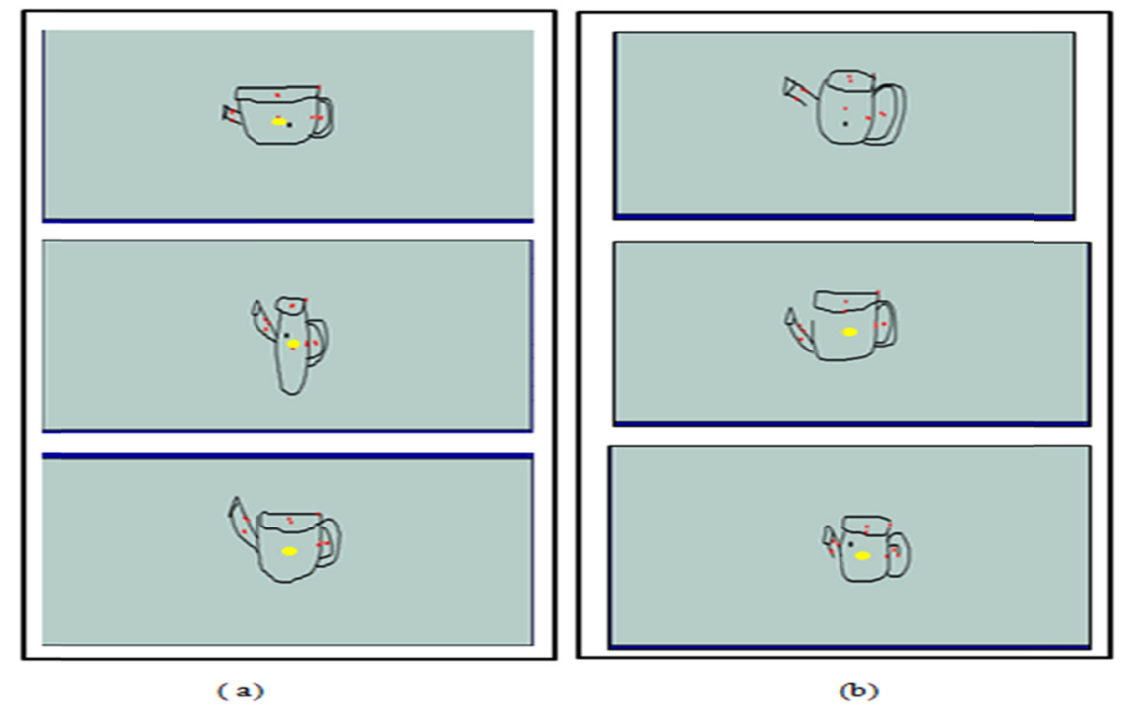

Figure 12. Example of Six teapot sketches (a) different types of drawing sketch teapot and (b) imperfect sketch

We noticed that each sketch related to the above six drawn sketches must contain seven attributes (northward curve, $\mathrm{x}$-axis eastward ellipse, westward curve, westward curve, northward-west line, northward-west line, and $\mathrm{y}$-axis westward ellipse), indicating the differences in drawn sketches.

\subsection{Dimensional Scaling}

Scaling can be defined as reducing or enlarging an object in comparison to the regular size (Scholz, 1986). Another definition of scaling is the ratio of the length in a drawing (or model) to the length of the real thing (Gansner et al., 2006). In previous studies, the problem of scaling has been solved in small dataset only by calculating the angle of sketch and comparing them with the angles of an object in an image. However, this issue is still unsolved in large datasets because a large dataset contains huge images that cannot be compared with angles of sketch (Riemenschneider et al., 2010; Cao et al., 2011). This problem has been solved in this study by depending on non-dimensional concept rather than dimensional by calculating the elements that comprise the 
sketch and extracting the number of elements for each sketch, so that the sketch can be recognized in different sizes depending on extracting the element composite of the sketch and retrieving the image property. To clarify the ability of the proposed method to solve the scaling problem, we draw two teapots as shown in Figure 12, we can noticed that, Figure 13 (a) shows sketch of a small teapot and (b) shows the same object drawn but larger size. The above mentioned successive steps of the proposal method are performed (1 to 4$)$ on each sketch. A vector of seven attributes (northward curve, $x$-axis ellipse, westward curve, westward curve, northward-west line, northward-west line, and y-axis ellipse) is also created that describe each sketch as a teapot regardless of the size (small or large) of the drawn sketch.

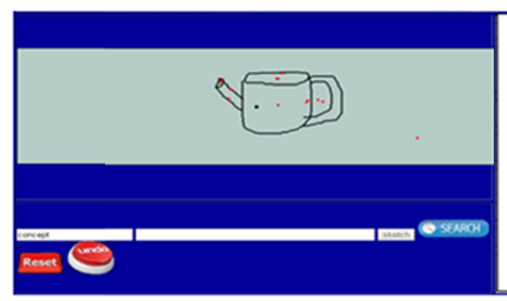

(a)

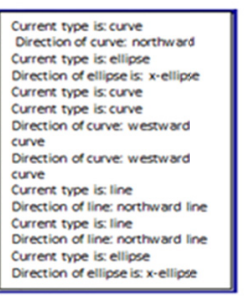
Current type is ellipse
Direation of ellipse is $x$-ellipse

Figure 13. Example of Scaling (a) small sketch, (b) large sketch

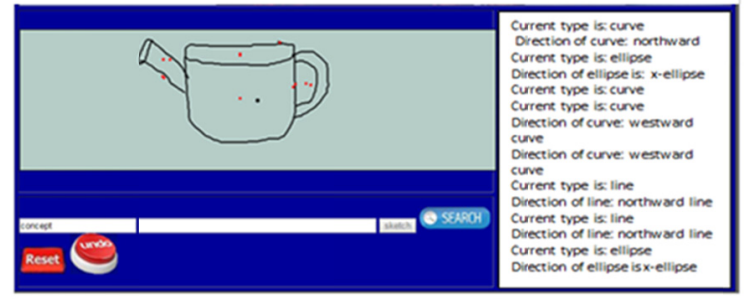

(b)

\subsection{Dimensional Transform}

The other essential issue in detecting the object for matching process is the object transform. Transform refers to moving the object in the workplace domain based on the same size, shape and orientation (Parui and Mittal, 2015; Hooshmand et al., 2012). This phenomenon came from the different user behaviour in drawing the sketch for search. To solve this problem in a sufficient way, the drawn sketch must ignore the positioning effect. For this reason, the presented method considers only sketch element effect. On the other hand, the selected object in the web images belonged to the metadata of the web, so it is not considered in the object position and it is concerned with the object name only as shown in Figure 14

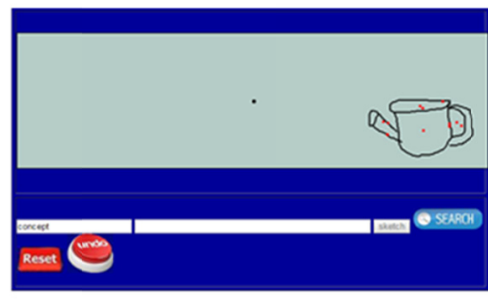

(b)

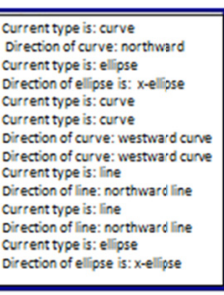

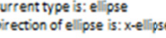

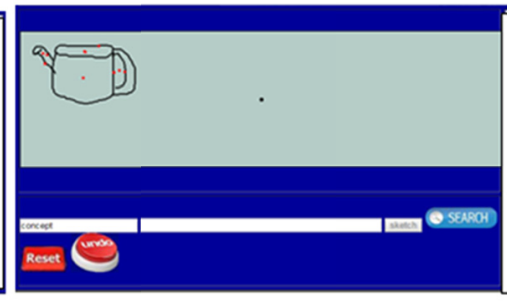

(a)

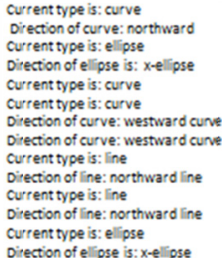

currection of ellipse is: $x$-ellipse

Figure 14. Example of Transpose (a) represent the location northward-west (b) sketch location southward-east

Figure 14 displays example of two teapots ( $a$ and $b$ ) drawn in the same area of drawing space. It is clarified that both sk4.etches have the same vector of attributes (northward curve, $\mathrm{x}$-axis ellipse, westward curve, westward curve, northward-west line, northward-west line, and y-axis ellipse), and the result will be teapots despite the different location of the drawn sketch in the sketch board space.

For each sketch entered in this system, this study has created a semantic space to save sketch attributes of sketches common input sketches. The stored sketch samples in MySQL are applied in the form of Semantic Space for sketch (M x N) matrix. The semantic spaces offer convenient methods to represent semantic relations between words and concepts. To create the basic mapping function, the dimensions of the semantic space will be identified by extracting shape elements as a matrix as introduced in table 1 . The created matrix is then fed by a row of queries from different kinds of users to cover all the possibilities of the user's hand skills 
Table 1. Explain the templates related to two sketches, moon and pyramid

\begin{tabular}{|c|c|c|}
\hline ID template & Sketch Template & Metadata \\
\hline 1 & Circle & Moon \\
\hline 2 & westward line eastward line & Pyramid. \\
\hline 3 & westward line eastward line westward line eastward line & Pyramid \\
\hline 4 & westward line eastward line westward line westward line westward line eastward line & Pyramid \\
\hline 5 & westward line eastward line horizontal line eastward line & Pyramid \\
\hline
\end{tabular}

\section{Drawing Question Sketch Online}

In this step, the method will be used for implementation. Where queries (drawings of a sketch by user) are entered and the attributes of the drawn sketch are extracted to create a vector of words (attribute), this vector will be compared with each template saved in mysql data base to match with suitable template in the database. This is the first function of to finding the mean of the input sketch and is accomplished by implementing the following:

Step 1: input queries (new sketch)

Step2: perform algorithm (1 to 2 ) to extract the keyshape geometric elements of sketch and make it a vector of words (attribute) as an input sketch.

Step3: match the template vector with all templates saved inMysql database.

Step 4: find the most similar template in the database.

Step 5: capture the metadata of the similar template with query template.

\section{Semantic Matching}

Semantic matching is a technique used in computer science to identify the information which is semantically correlated (Chauhan et al., 2013), In this process the exact match retrieval techniques provide a basic token matching capability in that only the documents that exactly match with the specified query can be retrieved consisting of one word or several words, considered as an abstract unit, and applied to a family of words related by meaning.

\subsection{Similarity Matching}

Matching is a technique used in computer science to identify the information that is a similarity correlated (Aziz and Rafi, 2010). In this step, the exact match retrieval technique that provides a basic matching capability is the document image metadata annotation that exactly matches the specified query that can be retrieved, consisting of one or several words.

Many methods have been proposed to match the sketch with images in dataset by using the low-level features process in order to make the image look like a sketch and match it with the sketch to be retrieved. This method has a good result when dealing with a small or limited database and retrieved images offline, but is not suitable for large database (Parui and Mittal, 2015; Bozas, 2012).

In this study, a new approach has been proposed to match the images with sketch in a high-level domain. That means understanding and recognizing the sketch meaning by analysing the geometrical function of the sketch, in order to return images based on that. In this step, it is important to mention that the matching was done using the process is presented in two tasks as below:

1. Linear pattern Similarity matching: this matching is performed between the vector of word (template) coming from users with database templates to find more vectors similar to it.

2. Matching with Google Engine: this matching is performed between the metadata captured from the (query) and submitted by URL engrained to be matched with the metadata annotation in Google images.

Linear pattern Similarity matching. It is important to clarify that mathematical similarity approach (Cosine similarity based on dot product) will be used to match the template of input vector with all templates saved database and to get the metadata related to input query.

The linear pattern similarity matching is used for matching the template of elements that came from the query with a template(s). Here, the cosine linear algorithm is used to measure the distance similarity between the template (query) with each template saved in mysql of two vectors (query and template stored). This measure is 
obtained by using Equations 7 and 8 to find the similarity between the vectors elements of the query with the vectors of template saved in the database under concept class. Cosine similarity based on dot product will present the similar template by choosing the highest rank of the saved query vector content that is stored as a string in the database.

$$
\begin{gathered}
\operatorname{sim}(X, Y)=X \bullet Y=\sum_{i} x_{i} \times y_{i} \\
\cos \theta(X, Y)=\frac{X \bullet Y}{\|X\| \times\|y\|}=\frac{\sum_{i}\left(x_{i} \times y_{i}\right)}{\sqrt{\sum_{i} x_{i}^{2}} \times \sqrt{\sum_{i} y_{i}^{2}}}
\end{gathered}
$$

Where $(\theta)$ refers to the angle between two vectors and $x_{i}, x_{j}$ are $n$-dimensional vectors. The resulting similarity ranges from 0 (exactly opposite) to 1 (exactly the same). In-between values indicate intermediate similarity or dissimilarity.

Algorithm 6 explains the sequence operation of similarity matching between the input query and the templates saved, by calculating the dot product and finding the exact template depending on the highest cosine $(\theta)$ value. Once the similar template is found based on the query and the metadata of this query is captured, the metadata is sent to URL Google images to retrieve the images related to the requested metadata.

Algorithm 6: Explains the sequence operation of similarity matching

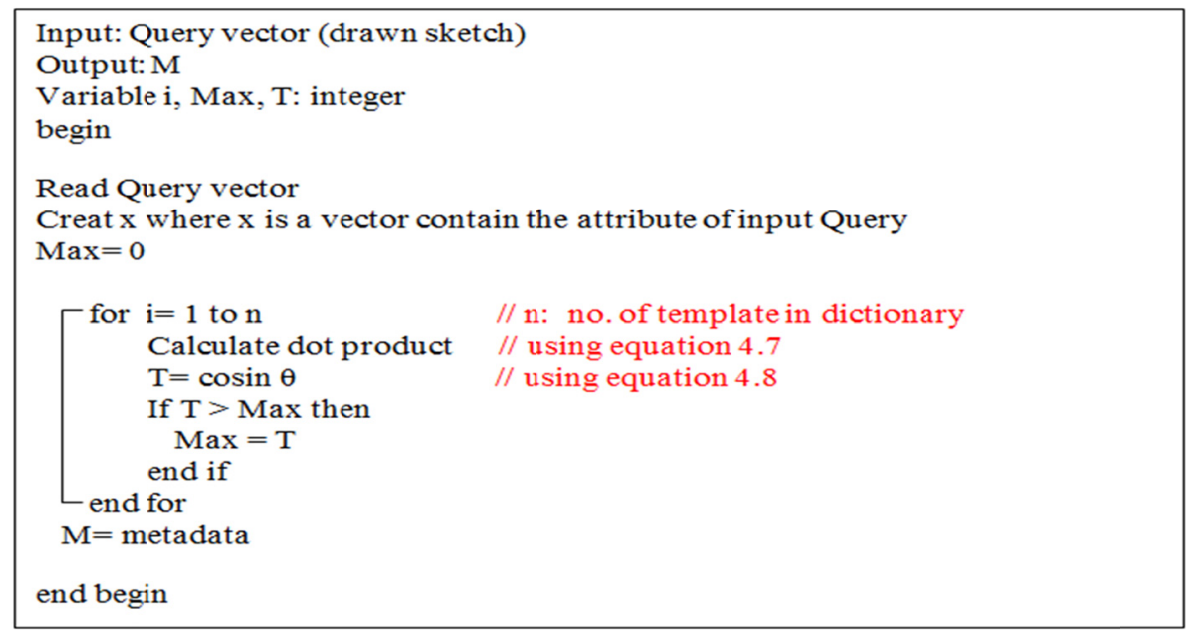

\subsection{Semantic Matching Based - Annotation}

It is important to mention that most of the traditional and common methods of image retrieval utilize some method of adding metadata such as captioning, keywords, or descriptions of the images so that retrieval can be performed over the annotation words. The dynamic progress in web images is used to generate new tools called automatic annotations for image control and management (Pramitasari et al.,2009). Taking advantage of automatic annotation in CBIR, the current approach functionally selects this method for retrieving sketch-based images. Furthermore, steps such as matching, annotation of image description in natural language of the content of the image in the Google engine repository and the conceptualizing the sketch semantically in a conceptual semantic domain which represent the query state is performed. The process sends the sketch to the web to find a similar expression in image annotation because each annotation includes a semantic description of each object in the image. This feature represents the best and the newest matching semantically is dependent on semantic conceptualization taken of the sketch with the semantic concept relevant to each image in the Google repository depending on annotation, Algorithm 7 is applied to find relevant images returned according to the concept of the query in web repository also Fig.8. Show examples of on the sketch Matching after achieved the concept of sketch. 
Algorithm 7: Search by image annotation

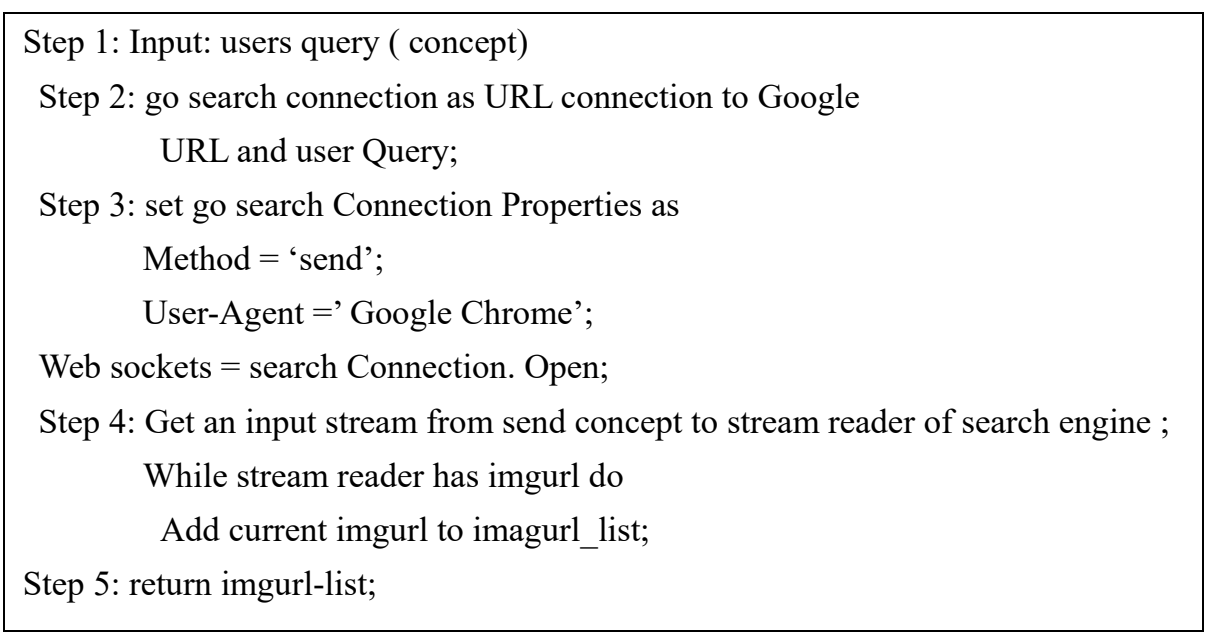

\section{Results and Discussion}

The sketch is conceptualized and used to retrieve the images from the web, which is based on the image automatic web annotation (Google images, flicker). Ten free hand sketches are used to retrieve the images from the web. Our method is tested by using the first page contain 30 images appeared on the search of each sketch, which are drawn on the sketch board online. The annotation features are used to acquire all the information, which can be used as a matching tool for the query to create an ontological form representing the sketch concept. The performance of the proposed method is illustrated in Fig. 15. The top five retrieved images for six input sketches are shown. The precision is evaluated by recalling the corresponding images for each sketch after retrieval. The sketch is conceptualized and a ranking score calculated for each image by precision@10 and achieved rate within 0.87 to 0.99 . Fig. 16, shows the precision-recall graphic compared to BoF, Key shape-based and Keyshape+BoF approaches (Saavedra, and Bustos, 2014). This evaluation shows that the performance of the keyshape mining based proposal increases the retrieval effectiveness, which satisfy correctly identified images True Positive (TP) for accuracy of retrieved images. This implies that all the images are retrieved. Moreover, the sketch specification is easy for transforming into the general ontological concept in a way that reduces False Negative (FN) images. To provide easy comparisons on a standard dataset and compute the recall which is difficult for a large dataset, we tested our system by using the same sketch shapes belonging to (Eitz et al., 2011). The Mean Average Precision (MAP) compared with the key shape base achieved (0.85639) where (keys-shape +BOF) achieved (0.88722) (Saavedra, and Bustos, 2014), and our method of mining the keyshape achieved (0.90422). Our proposed system not only performed accurately, but time is not a concern in retrieving depending on the network connection speed.

User dissatisfaction appears from the simple geometrical shape (set of lines, circle, or ellipse) used to draw the sketch. However, it is evident from the figure that when the shape elements become more complex the recall becomes higher and the precision is reduced. This results in the usual response in terms of the appearance of many unwanted images. However, the retrieved images are more specific.

The sketch board has a set of attributes with specific types. The nature of each attribute can be defined through the sketch board edition. To create a new sketch board, the following types of attributes are activated to display a plain hand draw type and a sketch category value from a range of predefined values of sketch elements. Edit the category records by clicking on the corresponding cell on sketch board. Query uploads can display the retrieved image path directly from the web and edits multi-category records on the sketch board. 


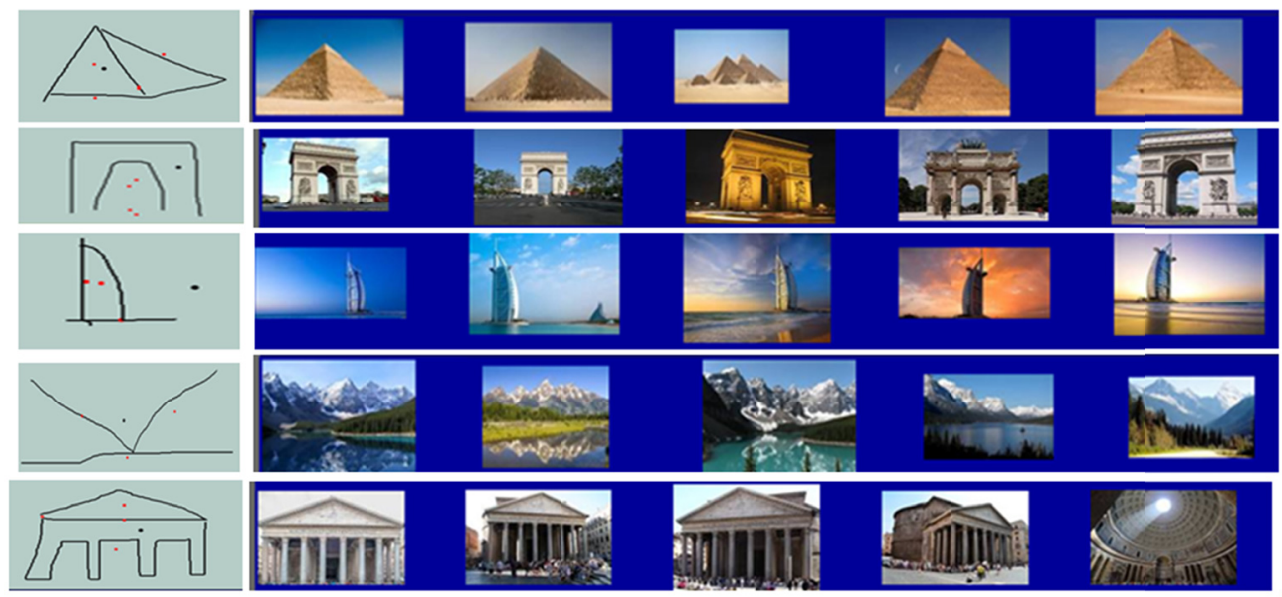

Figure 15. An example of SBIR using our proposal after retrieval images online. First column displays the hand drawing a sketch (query) by sketch board mining, and the next five columns show the first five images retrieval online in ( 3 Augest 2016)

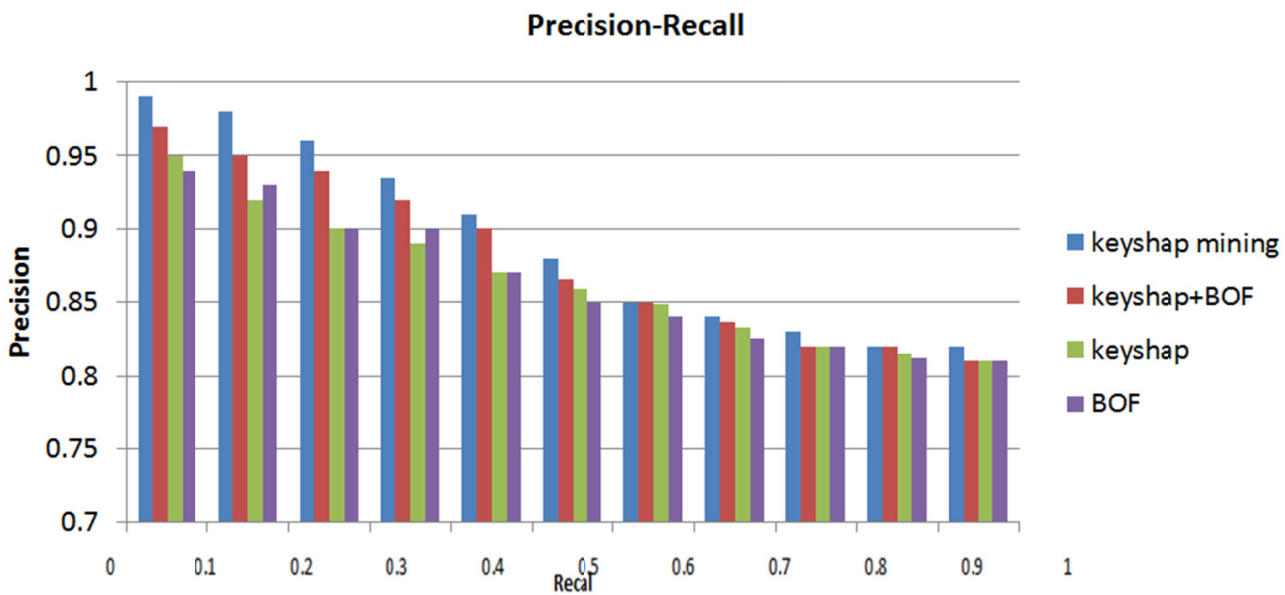

Figure 16. Precision and recall plot corresponding to the image for each sketch

\section{Conclusion}

This paper reports the sequence of process related to a sketch board mining. Mining represents the base of incoming drawing for interpreting the shape elements according to the Basic geometric elements (line, circle... etc.). They deal with conceptualized forms before the element is tied up with a spatial relationship to ensure the dynamic state of the sketch as a mimic to user drawing. The process guides us to reach high conceptual levels (semantic level) of the object to accomplish the specific annotation of the object based on ontology, which represents the query. The annotation of query enables us to match the sketch concept with the specific meaning of the web images (annotation) representing the highest level of retrieval accuracy. Global repository images are retrieved through a semantic match of the user's sketch query. A recall rate in the range of 0.1-0.8 and a precision rate between 0.78-0.97 are achieved, the retrieval effectiveness achieving an MPA 0.90422. The advantage of our innovative method is to draw the sketch in real time and take the possibilities of different users by determine the different element of sketch depending on the measure distance between the center and the tangent of element (which represent the threshold of the element drawing) that make us solve many problems like determining the arc from the line drawing inaccurately and to determine the direction of concavity and convexity of the curve. However, results need further improvement in terms of precision-recall to specify the retrieved images according to conceptual based mining techniques. It is asserted that the present technique is able to exploit high-level features to search the web efficiently and attractive for accurate image retrieval. 


\section{Acknowledgements}

Huda Abdulaali would like to thank the Ministry of Higher Education \& Scientific Research- (Al-Mustansiriya University), College of Science, Computer Department Iraq and UTM-IRDA Digital Media Centre (MaGIC-X), Faculty of Computing, Universiti Teknologi Malaysia, Malysia.

\section{References}

Abdulbaqi, H. A., Sulong, G., \& Hashem, S. H. (2014). A sketch based image retrieval: a review of literature. Journal of Theoretical and Applied Information Technology, 63(1).

Aggarwal, C. C. (2007). Data streams: models and algorithms (Vol. 31). Springer Science \& Business Media.

Aziz, M., \& Rafi, M. (2010, August). Sentence based semantic similarity measure for blog-posts. In Digital Content, Multimedia Technology and its Applications (IDC), 2010 6th International Conference on (pp. 69-74). IEEE.

Banfi, F. (2000). Content-based image retrieval using hand-drawn sketches and local features a study on visual dissimilarity.

Boolos, G. S., Burgess, J. P., \& Jeffrey, R. C. (2002). Computability and logic. Cambridge university press.

Bozas, K., \& Izquierdo, E. (2012, July). Large scale sketch based image retrieval using patch hashing. In International Symposium on Visual Computing (pp. 210-219). Springer Berlin Heidelberg.

Cao, Y., Wang, C., Zhang, L., \& Zhang, L. (2011, June). Edgel index for large-scale sketch-based image search. In Computer Vision and Pattern Recognition (CVPR), 2011 IEEE Conference on (pp. 761-768). IEEE.)

Chauhan, S., Arora, P., \& Bhadana, P. (2013). Algorithm for semantic based similarity measure. Int. J. Eng. Sci. Invent, 2(6), 75-78.

Dube, D., \& Zell, A. (2011). November. Real-time plane extraction from depth images with the randomized hough transform. In Computer Vision Workshops (ICCV Workshops), 2011 IEEE International Conference on (pp. 1084-1091). IEEE.

Eitz, M., Hildebrand, K., Boubekeur, T., \& Alexa, M. (2011). Sketch-based image retrieval: Benchmark and bag-of-features descriptors. Visualization and Computer Graphics, IEEE Transactions on, 17(11), $1624-1636$

Eitz, M., Richter, R., Boubekeur, T., Hildebrand, K., \& Alexa, M. (2012). Sketch-based shape retrieval. ACM Trans. Graph., 31(4), 31-1.

Funkhouser, T., Min, P., Kazhdan, M., Chen, J., Halderman, A., Dobkin, D., \& Jacobs, D. (2003). A search engine for 3D models. ACM Transactions on Graphics (TOG), 22(1), 83-105.

Gansner, E., Koutsofios, E., \& North, S. (2006). Drawing graphs with dot (pp. 1-17).

Guarino, N., \& Welty, C. (2000, August). Identity, unity, and individuality: Towards a formal toolkit for ontological analysis. In ECAI (Vol. 2000, pp. 219-223).

Gupta, S., Kumar, D., \& Sharma, A. (2011). Data mining classification techniques applied for breast cancer diagnosis and prognosis. Indian Journal of Computer Science and Engineering (IJCSE), 2(2), 188-195.

Hooshmand, A., Campbell, M. I., \& Shea, K. (2012, August). Steps in transforming shapes generated with generative design into simulation models. In ASME 2012 International Design Engineering Technical Conferences and Computers and Information in Engineering Conference (pp. 883-892). American Society of Mechanical Engineers.

McManus, I. C., Chamberlain, R., Loo, P. W., Rankin, Q., Riley, H., \& Brunswick, N. (2010). Art students who cannot draw: Exploring the relations between drawing ability, visual memory, accuracy of copying, and dyslexia. Psychology of Aesthetics, Creativity, and the Arts, 4(1), 18.

Mendelson, E. (2009). Introduction to mathematical logic. CRC press.

Parui, S., \& Mittal, A. (2014). Similarity-invariant sketch-based image retrieval in large databases. In Computer Vision-ECCV 2014 (pp. 398-414). Springer International Publishing.

Pramitasari, L., Hidayanto, A. N., Aminah, S., Krisnadhi, A. A., \& Ramadhanie, A. M. (2009). Development of student model ontology for personalization in an E-learning system based on semantic web. InInternational Conference on Advanced Computer Science and Information Systems (ICACSIS09), Indonesia, December (pp. 7-8). 
Riemenschneider, H., Donoser, M., \& Bischof, H. (2011, February). Image retrieval by shape-focused sketching of objects. In 16th Computer Vision Winter Workshop (p. 35).

Saavedra, J. M., \& Bustos, B., 2014. Sketch-based image retrieval using keyshapes. Multimedia Tools and Applications, 73(3), 2033-2062.

Scholz, C. H., Aviles, C. A., \& Wesnousky, S. G. (1986). Scaling differences between large interplate and intraplate earthquakes. Bulletin of the Seismological Society of America, 76(1), 65-70.

Sulong, G., Abdulaali, H., \& Hassan, S. (2015). Edge Detection Algorithms VS-active Contour for Sketch Matching: Comparative Study. Research Journal of Applied Sciences, Engineering and Technology, 11(7), 759-764.

Tencer, L., Režnáková, M., \& Cheriet, M. (2012, July). A new framework for online sketch-based image retrieval in web environment. In Information Science, Signal Processing and their Applications (ISSPA), 2012 11th International Conference on (pp. 1430-1431). IEEE.

Weinbrenner, S., Engler, J., Tehrani, P. F., \& Hoppe, H. U. (2012, March). Semantic Interpretation and Feedback for Digital Sketching Environments. InWireless, Mobile and Ubiquitous Technology in Education (WMUTE), 2012 IEEE Seventh International Conference on (pp. 223-225). IEEE.

Zhou, R., Chen, L., \& Zhang, L. (2012, October). Sketch-based image retrieval on a large scale database. In Proceedings of the 20th ACM international conference on Multimedia (pp. 973-976). ACM.

\section{Copyrights}

Copyright for this article is retained by the author(s), with first publication rights granted to the journal.

This is an open-access article distributed under the terms and conditions of the Creative Commons Attribution license (http://creativecommons.org/licenses/by/4.0/). 